\title{
Flexible COM - based software solution for HFC Network Performance monitoring
}

\author{
Hung Nguyen Chan Ph.D. student, Judith Redoli Granados Ph.D., Rafael \\ Mompó Gómez Ph.D., Belen Carro Martinez Ph.D. student.
}

Department of signal theory and Telematic Engineering - ESTI, University of Valladolid

47011 Valladolid - Spain.

Abstract: $\quad$ Emerging HFC (hybrid fiber coaxial) networks with activated return path for data communications requires careful performance monitoring and preventative maintenance over the entire network. Return path is especially problematic due to the noise funneling effects that can strongly affect digital services. Besides, the HFC networks which base on existing CATV infrastructures require many considerations. The special characteristics of HFC network lead to the needs of taking into account many issues related to software, hardware, measurements, etc. This article focuses on the designing issues of HFC network monitoring software to deal with the noise problem in the physical layer, in respect to the trends of measurement software, and describes a performance monitoring software framework, which applies the Component Object Model (COM) approach. The software has been developed in the CEDETEL Lab - ESTI, University of Valladolid, in collaboration with RETECAL - Spain. The article is organized as follows: the first section addresses the general problems of HFC performances. The second section presents the typical issues of monitoring the performance of HFC physical layer. In the third section, the key issues of designing software for HFC network monitoring, and the COM approach will be discussed. In the fourth section, an HFC performance monitoring application, which has been developed in respect to the issues mentioned on the previous sections will be described. Finally, the last section presents conclusions and future works.

The original version of this chapter was revised: The copyright line was incorrect. This has been corrected. The Erratum to this chapter is available at DOI: 10.1007/978-0-387-35522-1_37 
Keywords: CATV networks, hybrid fiber coaxial, performance monitoring, physical layer, software, COM.

\section{INTRODUCTION}

An HFC network is a communication network in which a variety of telecommunication systems coexist. The most critical problem of the network is the noise and ingress in the return path. The noise is produced by many sources, such as TVs, Set-top Boxes, and other electrical domestic devices. Due to the noise funneling effect, the noise introduced by one subscriber can influence the network performance and service qualities through a wide region. In other words, a strong source of noise from one subscriber's home can affect other subscribers. That is why HFC networks change over time and need periodic measurements to maintain proper performance. Each time a new user gets into the network, the characteristics of the shared medium are modified. This changing medium depends mainly on the users themselves. That fact leads to the important role of the preventative maintenance program, which includes the status monitoring to focus maintenance efforts. The monitoring system should be able to automatically measure and collect the most relevant parameters of both downstream and upstream channels such as vision carrier level, sound carrier level, C/N (Carrier/Noise), CTB/CSO (Composite Triple Beat / Composite Second Order), for digital service: BER (Bit Error Rate), EVM (Error Vector Magnitude), etc. Technicians will consult these results to detect network problems. In the case that the problem already has occurred, measurement data also should be reviewed and compared with the previous data. Only the most critical places in the network will be visited and tuned. (BARCO [14]). Therefore, some monitoring equipment should be placed in the important points such as the headend and distribution centers. Different equipment should be dedicated for different kinds of measurements.

As the number of measuring points is high due to the structure of the network, automatic measurements and a properly defined data base are necessary to control an adequate performance of the HFC network.

So far, the HFC network is not strictly standardized. Currently, the DOCSIS, IEEE 802-14, DAVIC/DVB standards are coexisting. Several vendors even develop products of their own technical specifications. In most of the cases, upgrading for data service requires a complete solution from a specific vendor including headend, cable modem termination system (CMTS), cable modems and software [23]. There are several software solutions on the market to monitor noise and ingress on the HFC network ([11] and [24]). Their common drawback is that they only support a small set 
of hardware from specific vendors. These facts raise the need of developing a flexible solution for monitoring HFC access network, which can handle a large set of instruments, support hardware/software reusing, and easily be adapted to the future needs.

\section{PHYSICAL LAYER PERFORMANCE ISSUES}

So far, many trials and models have been developed to study the effects of noise and interferences to HFC network. Some laboratory models of Motorola, General Instruments, and CableLabs were described in the Joyce [6], Eldering [7] and Williams [16].

In the operating network, it is required to automatically monitor performance as well as quickly detect problems with minimum affect on normal service. The hardware requirements and measurement procedures were covered in detail in Thomas [1] [2], Bill [15], Feather [17], Parker [18] and NCTA [22].

In general, both models have the similar configuration, which is illustrated in Figure 1.

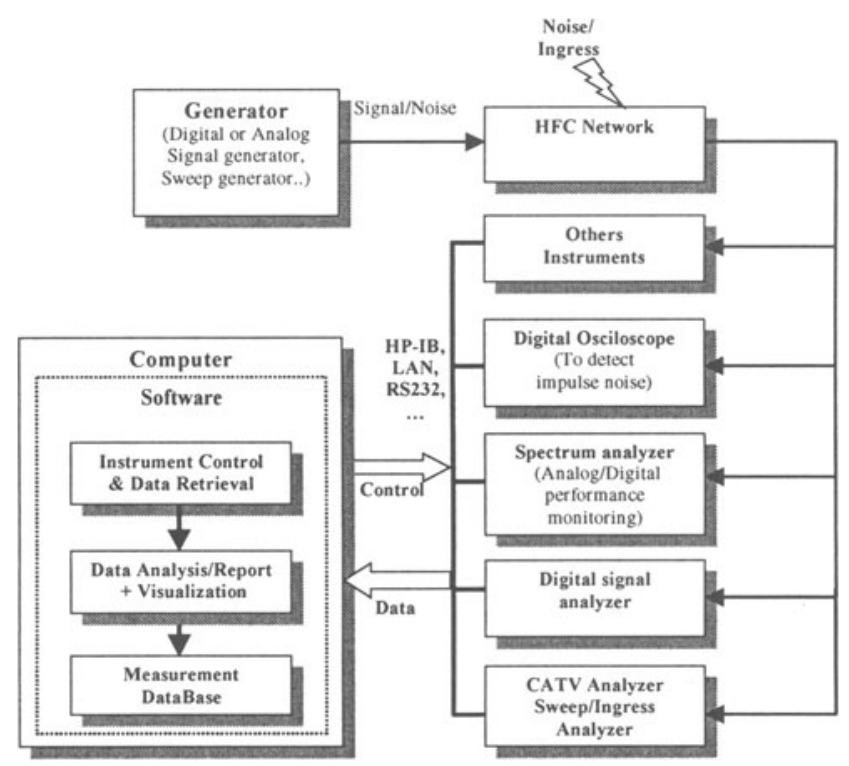

Figure 1.Typical configuration of monitoring performance of HFC physical layer.

As can be seen from the figure, the software should provide interoperability between various types of instruments (can be from various 
vendors). The second thing can be seen is that the laboratory model can be adapted to work in the real network because of their similarity.

\section{DESIGNING ISSUES OF HFC PERFORMANCE MONITORING}

\subsection{Measurement issues}

As mentioned in previous sections, measurement functions play the most important role in HFC performance monitoring because of its use to combat with noise/ingress problem and observe digital/analog performance. The designing issues of measurement software will be discussed thoroughly in this section.

\subsubsection{Background}

The measurement software stack is illustrated in Figure 2.

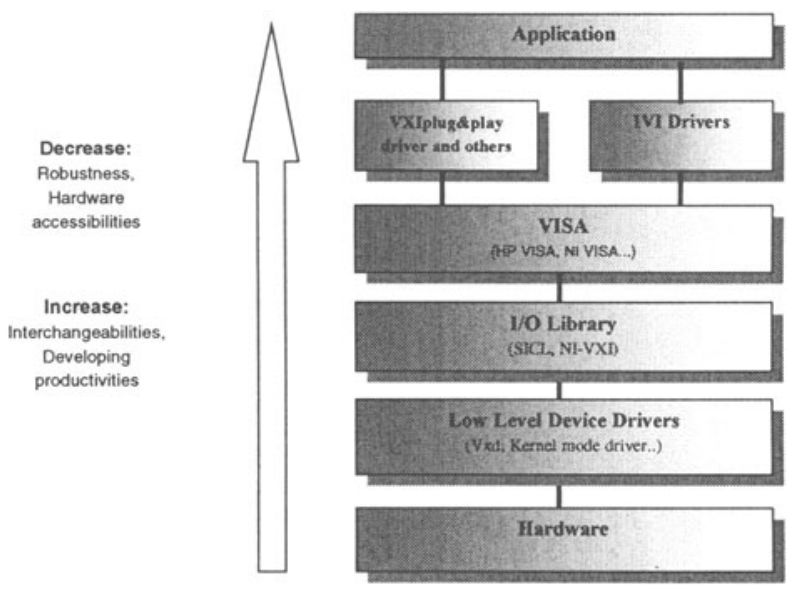

Figure 2.The measurement software stack

The goals of introducing several layers in the software model are:

- Providing compatibilities between various instrument vendors. 
- Increasing the interchangeabilities between vendors and hardware/firmware versions.

- Reducing the programming efforts of measurement software developers by eliminating the needs of accessing low-level drivers/firmware.

More details can be found in the article of Rowe and Kerridge [19].

\subsubsection{New trends of measurement software}

Recently, measuring software is moving toward the interchangeabilities between vendors and hardware/firmware versions. In other words, it is desired that the specific measurement software not be changed when the hardware is replaced. To achieve that, one layer must be added to the legacy measurement software model. This new layer, known as Interchangeable Virtual Instrument (IVI) drivers, provides the interchangeabilities for the software model. If the hardware is replaced, only IVI drivers would be changed and the application should be kept unchanged.

So far, there are not yet standards for the IVI drivers. The IVI foundation [21] is still working on the drafts. There are many possibilities that IVI drivers will be based on Component Object Model (COM) technology of Microsoft due to the following reasons:

- COM is directly supported by MS Windows OS and the most popular developing tools like MS Visual C++, Java, MS Visual Basic, Borland Delphi, etc.

- The new emerging developing tools, known as Rapid Application Development (RAD), such as MS Visual Basic and Borland Delphi, are based on COM/ActiveX technology. Therefore, supporting COM can greatly reduce developing time by making use of these tools.

- COM is now available on most major platforms. Therefore it can provide interoperability between various types of computer hardware running on various operating systems ([25], [26]).

- $\mathrm{COM}$ has become an industrial standard.

- A great number of COM-based software components, known as ActiveX components, have been offered by most of software vendors. Therefore using COM will greatly take advantage of software reuse.

- COM provides the location and platform transparency. [20]

Oblad [3] and Fertita [4] studied deeply the benefits of implementing COM into Test\& measurement industry. The previous efforts of VXIplug\&play Systems Alliance led to the introduction of VXIplug\&play C-based drivers (to support multi-platform hardware and software). 
However, together with the rapid progress of Information Technology, this approach is recently found inappropriate.

Other competitive software component technology, such as CORBA (COM Object Request Broker), is not considered adequate due to the far less popularity in Test and Measurement filed. However, the IVI foundation is considering the possibility of allowing migration into CORBA and other technologies [27].

\subsubsection{Location independence}

To achieve location independence between hardware equipments, several possibilities can be implemented:

- GPIB LAN gateway: the measurement instruments will connect to a gateway (a computer or dedicated hardware), which has a LAN interface.

- Web Server: the measurement software has a built-in Web server that allows remote user access using popular Web navigators.

- Users can connect to measurement software on remote servers through communication software components.

- The instrument drivers based on DCOM (Distributed COM), which has remote capabilities, or in other words, location transparency. This option has been recommended for future IVI drivers.

\subsection{Data analysis}

The laboratory models require powerful data analysis and visualization tools such as MATLAB, while for real network reports, MS Office documents are usually used. In both cases, applying COM technology is very beneficial since MATLAB, MS Office and many other popular software tools support OLE automation (COM-based).

\section{APPLICATION SOFTWARE FRAMEWORK FOR HFC PERFORMANCE MONITORING}

\subsection{Designing issues and their implementations}

Taking into account the above-mentioned issues, a software framework has been developed to support HFC performance monitoring in the laboratory environment and easily adapted to the demand of real networks. 
The application, named "Magic Monitor for CATV", runs in the MS Windows 9x/NT OSs, which are the most popular OSs at the moment.

Figure 3 is the software block diagram of the software framework. Designing issues and their implementations will be discussed next.

\subsubsection{Developing tools}

In order to become a high performance multi-threaded application, the framework was written by MS Visual C++ and implement MFC (Microsoft Foundation Classes).

Several software components, such as software agents (client and server side) were written in Visual Basic in order to reduce developing time.

The interface between the software components and the main application were written using ATL (Active Template Library), which simplifies COM programming.

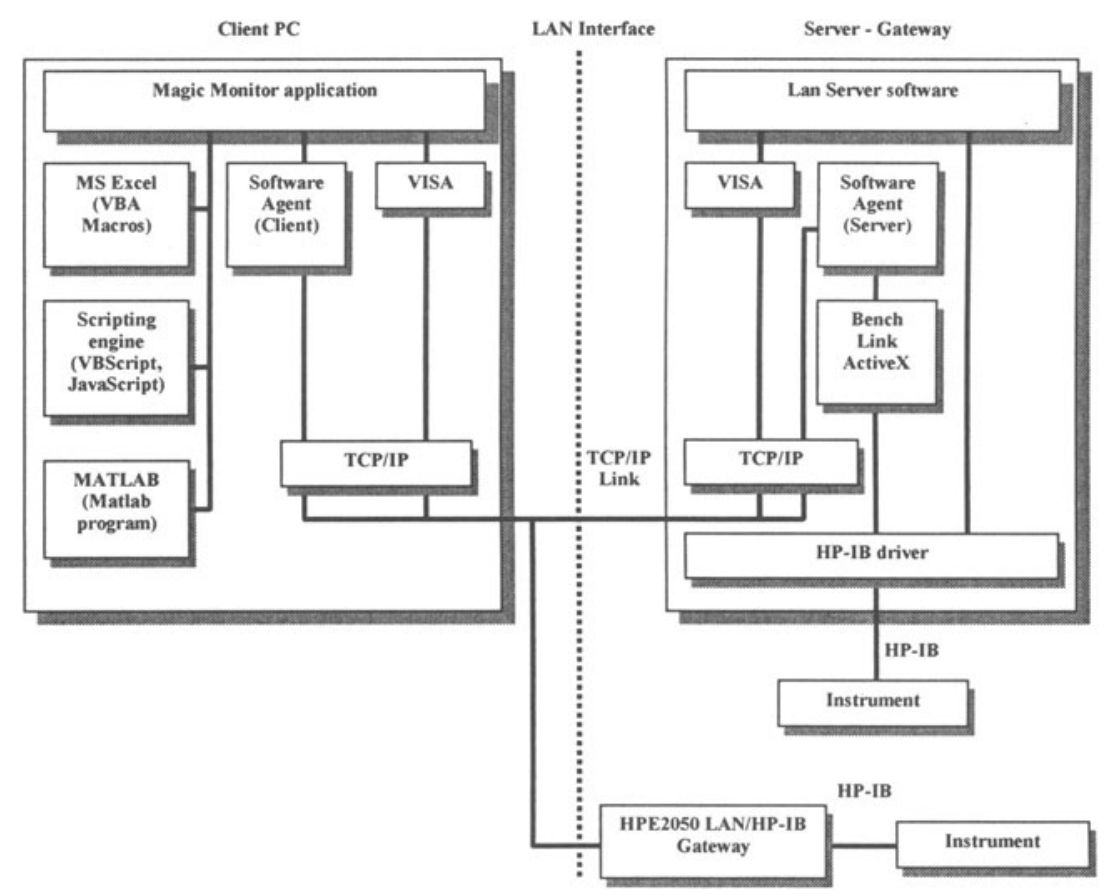

Figure 3. Software block diagram of the "Magic Monitor" framework 


\subsubsection{Software component based}

Instead of building a fixed-function application, it has been broken down into many small components to increase flexibilities.

As Brockschmidt [20] indicated, one of the strongest features of COM is software component reuse. Therefore, instead of rewriting a feature for the application, it is better to connect to a software component (ActiveX), which already has this feature, through COM interfaces. This approach has been thoroughly implemented in "Magic Monitor".

\subsubsection{Scripting and macro language support}

Scripting \& Macro languages such as Visual Basic for Application (VBA) and VBScript strongly support COM.

Therefore, Magic Monitor provides the simple Application Development Environment (ADE) for creating, editing, and debugging simple macros that can launch other scripts such as VBA program and VBScript in a new thread.

Writing macros and scripts is rather simple. In order to write a VBA macro, users can simply record a macro in MS Office, then optionally edit it in the VBA developing environment, which goes together with MS Office.

Supporting scripting language has a great advantage because of the introduction of the new MS Window Scripting Host technology [28], [29]. This new technology allows automating OS tasks by executing VBScript and JavaScript directly in Windows environment and controlling ActiveX-based software via COM interfaces. The power of scripting \& macro language allows them to directly handle instruments if their drivers are COM-based.

\subsubsection{Flexibilities}

The application itself took the idea of macro language: in order to do a task, write a macro, assign it to a button or menu, and do the task with just one mouse click. This approach provides great flexibilities, since once the task needs change; one just has to modify the macro. Magic Monitor macros will have the power of VBA, VBScript, JavaScript and MATLAB programs, since they can be integrated into the macros.

On the measurement side, Magic Monitor controls the VISA layer directly, and therefore, has the flexibilities of hardware access and robustness, but will also be able to handle future IVI drivers, which will be COM-based and intended to use in scripting/macro languages.

To facilitate macro writing, some solutions have been implemented. For example, users can select a command from a drop down list using mouse, the 
whole command will be automatically completed by the software, and added to macro text. The help system is also improved, as described in the next section. A macro usually contains from 10 to 200 lines, including user's comments.

The procedures to make monitoring tasks using the application are illustrated in Figure 4.

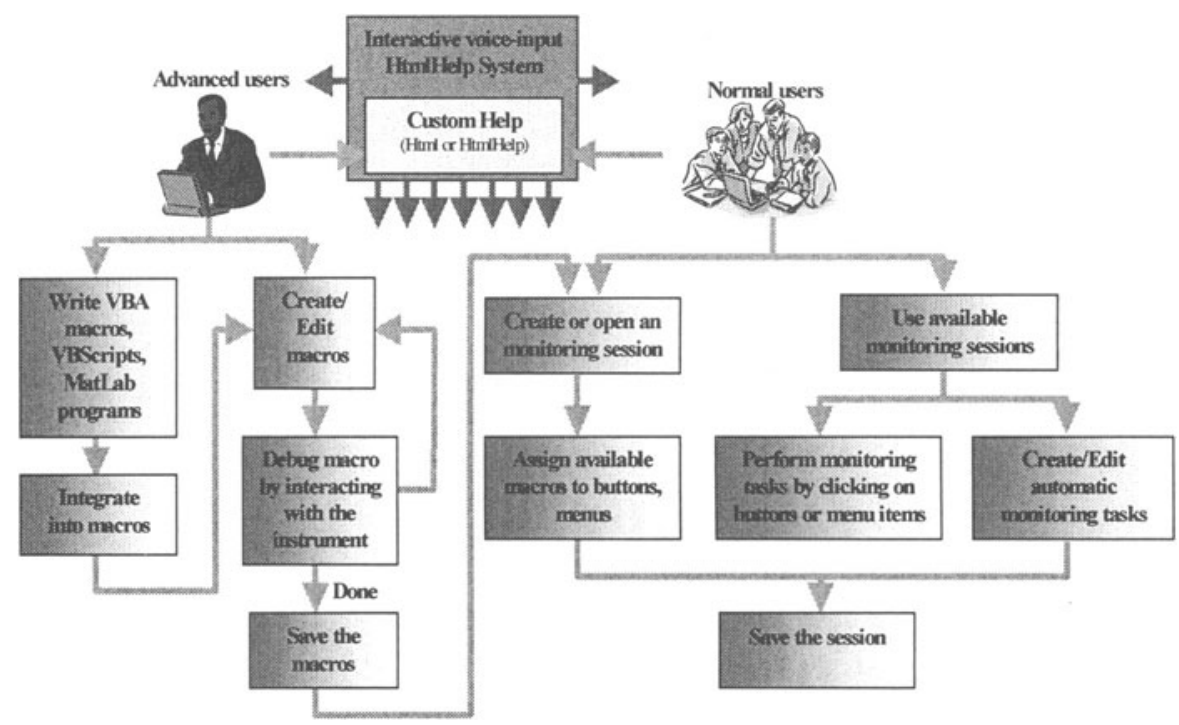

Figure 4.Creating monitoring tasks using Magic Monitor

\subsubsection{Improvement in Help tools.}

In our experiences, the roles of documentations and Help systems have increasing value as the amount of information expands, especially when facing programming and workgroup co-operating problems. Therefore, an advanced expandable $\mathrm{HtmlHelp} / \mathrm{Html}$ system has been developed to assist group users/programmers. The new MS HtmlHelp system has the full-text Boolean search capability, very much like Internet search mechanism, can effectively facilitate programming and using software. Besides, the help system was designed to easily expand. Should a user write a new macro, he or she can also write its documentations and integrate it into the global help system. The new MS Agent and speech technologies (speech synthesis and recognition) are also made available as an option. So far, speech engines are available in all major languages. Therefore, the speech-enable interactive help system will have increased values. 


\subsubsection{Location independencies}

The application can run locally as well as remotely. It has several remote options:

- Remotely control instruments via a small-size dedicated hardware HP- E2050 gateway, which is very convenient for remote operating network monitoring.

- Control instrument via a PC, which works as a measurement server. In this case, client PC can also view the real instrument image screen by controlling the BenchLink ActiveX component via two software agents. Figure 5 is the application's screenshot.

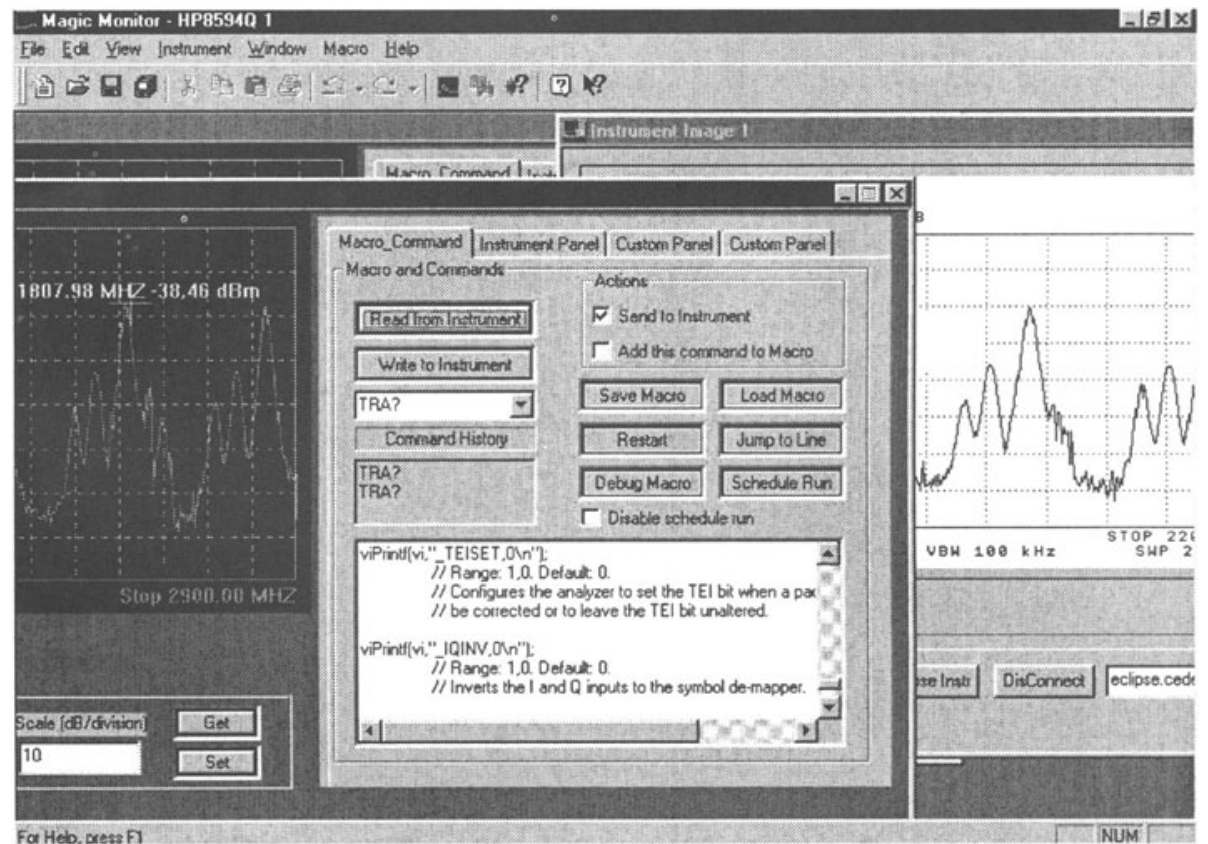

Figure 5.Magic Monitor Screenshot

\subsection{Project Results}

\subsubsection{Software capabilities:}

- Providing the capabilities to perform automatically scheduled remote measurement tasks on most of the programmable instruments such as spectrum analyzers, oscilloscopes, arbitrary/function generators, etc 
through GPIB and RS232 interfaces and collect the measuring data into files.

- Remotely retrieving screen images of various instruments and saving them in compressed formats.

- Having the advanced expandable voice-interactive Help System to assist users to write macros and perform monitoring tasks.

- Performing data analysis automatically from the application environment by executing VBA macros.

- Executing VBScript, JavaScript and MATLAB programs from inside macro.

- Dynamic Graphical User Interface (GUI): users can create their new buttons and menu items to the GUI, link them to macros and perform tasks simply by mouse.

- Allowing automatic customizable CATV measurements with standard spectrum analyzers.

- Providing easy-to-use application development environment (ADE) to write and edit macros.

\subsubsection{Applications}

Based on the software framework, a research model has been constructed in the CEDETEL CATV Laboratory - University of Valladolid, in collaboration with RETECAL, a northern Spain Cable TV operator. The main target of the project is studying the digital service aspects in Spanish HFC network conditions and assisting CATV/HFC network OAM\&P. The model is shown in Figure 6.

The goal of the model is comparing and analyzing measurement data from the CATV Lab trial (CEDETEL - Spain), the operating network of RETECAL, and the simulation model. The automatic data retrieving and analyzing are integrated and synchronized by using the software framework. 


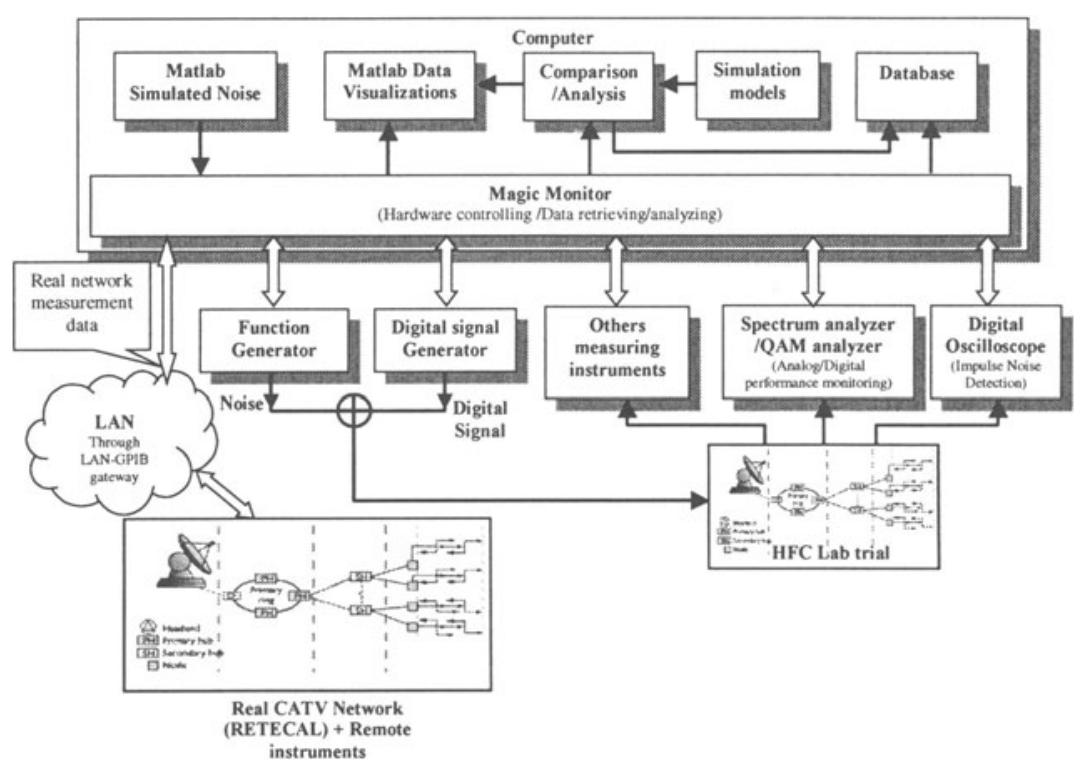

Figure 6.Hardware/software model to study HFC performance applying the "Magic Monitor" framework.

\section{CONCLUSION AND FUTURE WORKS}

This article has addressed a several issues of design and implementation of HFC performance monitoring software to deal with the noise problems in the physical layer, regarding the latest progresses in Information Technology and Test \& Measurement trends. The measurement problems have been highly focused on.

An application that has been developed implementing the considering issues has been described as an example.

It can be concluded that COM technology is a promising candidate for the HFC performance monitoring software due to its power and popularity. This will cut down developing time as well as increase flexibilities and hardware/software interchangeabilities.

As HFC network is new and always subject to change, flexibility is one of the most important features that the monitoring software should have. 
Supporting COM, and macro/scripting languages are good options to increase the flexibilities.

With the current framework, we will develop more software components to serve our researching demands. The application in the real network and the integration of hardware-software simulation will be further studied.

\section{References:}

[1] Thomas J., Edginton.M, Digital Basics for Cable Television Systems; Prentice Hall 1999.

[2] Thomas J., Cable Television Proof of Performance: A complete guide to Cable TV Compliance Measurement using a Spectrum Analyzer, Prentice Hall 1995.

[3] Oblad R., Achieving robust interchangeability of Test assets in ATE systems, Proceeding AutoTestCon 1999. www.hp.com

[4] Fertita K., Harvay .J. The Role of ActiveX and COM in ATE, Proceeding AutoTestCon 1999. www.hp.com

[5] Bahatti S., Knight .G, On Management of CATV Full Service Networks: A European Perspective, IEEE Network September/October 1998. p28-39.

[6] Joyce G., Moran .J, Henderson .S Gaetani .D, The Ultimate Information Capacity of HFC Return Path, Motorola 1997. p4-6.

[7] Eldering C., Himayat .N, Gardner .F, CATV Return Path characterization for Reliable Communications, IEEE Communication magazine August 1995. Vol 33 , p62-69

[8] Sucharczuk G., Advanced Network management, International Cable ,October 1997. p 40.

[9] Fox S., Five steps to Reduced Return Path Ingress, International Cable, May 1998. p 62.

[10] Walter C., James F., David L., Modern Cable Television Technology, Morgan Kaufman Publisher, Inc.1999.

[11] Hewlett-Packard http://www.hp.com.

[12] National Instrument http://www.ni.com

[13] Microsoft Developer Network (MSDN) http://msdn.microsoft.com

[14] BARCO Communication Systems, Achieving accurate network monitoring in the cable environment to improve the level of service and reliability, http://www.barco.com/communic/products/Monitoring_Management/network/accuratenet monitoring.htm.

[15] Bill M., Noise and ingress performance in the return path; CED Magazine March 1997.

[16] Williams T., Plant architectures for diagnosing and maintaining bi-directional HFC network, SPIE proceeding November 1996. SPIE Vol.2917, p12.

[17] Feather B., Understanding Software standard, International Cable December 1997. p48 51.

[18] Parker D., Network management: The key to system reliability, International Cable. May 1997. p10-14.

[19] Rowe M., Kerridge B., Software drivers: What are they exactly?, Test and measurement World, March 1999. http://tmworld.com/articles/03 1999 Drivers_cover.htm

[20] Brockschmidt K., What OLE is really about, Microsoft Developer Journal July 1996, p43-45.

[21] IVI Foundation. http://www.ivifoundation.org/Operations/charter.htm

[22] National Cable Television Association, NCTA Recommended practices for measurement on CATV systems, Supplement on upstream issues, Oct 1997.

[23] Kiniry J., Mertz C., Cable Modems: Cable TV delivers the Internet, IEEE computing May/June 1998. p13.

[24] Tektronix www.tek.com

[25] Microsoft COM technology, http://www.microsoft.com/com/ 
[26] SoftwareAG solutions, http://www.softwareag.com/entirex/product/EntireXengl.pdf [27] IVI foundation meeting summary, Nov 1999 http://www.ivifoundation.org/Meetings/1999Nov/99NovMeetingSummary.doc, p.42. [28] MS Window script Technology, http://msdn.microsoft.com/scripting/

[29] MS Windows Scripting Host programmer's reference. http://msdn.microsoft.com/scripting/ 